\title{
ORIGINAL ARTICLE \\ Physiological responses between players with and without spinal cord injury in wheelchair basketball small-sided games
}

\author{
A Iturricastillo, J Yanci, A Los Arcos and C Granados
}

\begin{abstract}
Study Design: This is a comparative study between players with and without spinal cord injury (SCI) during a training task. Objective: This study examined physiological responses in commonly used small-sided games (SSGs) in well-experienced wheelchair basketball (WB) players with $\mathrm{SCl}$ and without SCI (Non-SCI).

Setting: The study was conducted with a WB team in Vitoria-Gasteiz, Spain (2014).

Methods: The team was divided into an $\mathrm{SCl}$ group $(n=6)$ and a Non-SCI group $(n=6)$. Absolute and relative heart rate $(\mathrm{HR})$ along with tympanic temperature and perceived exertion (RPE) were recorded for both groups.

Results: The two groups attained different absolute HR values for the same SSG. However, no significant differences were observed in relative HR between groups (\%HRmean, \%HRpeak and the percentage of the time spent in each HR zone: low, moderate, high and maximal) nor in tympanic temperature. Moreover, in relation to the bout evolution analysis (4 repetitions of 4 min), the Non-SCl group significantly increased $(P<0.05)$ absolute HRmean and HRpeak during bouts, whereas the SCI group maintained them constant. Furthermore, the variations in the percentage of the time spent in each HR zone only were observed in the Non-SCl group.

Conclusion: In spite of the Non-SCl group attaining higher absolute HR values, the SCl and Non-SCl groups may have similar HR relative values during a specific WB training task. However, the SCl group reported significantly higher values in respiratory RPE in the last bout than the Non-SCI group for the same SSG.
\end{abstract}

Spinal Cord (2016) 54, 1152-1157; doi:10.1038/sc.2016.43; published online 12 July 2016

\section{INTRODUCTION}

Physiological demands in team sports have been analyzed in many studies during matches ${ }^{1,2}$ and training sessions. ${ }^{3,4}$ However, there is a lack of information about the time spent performing activities specific to wheelchair basketball (WB) and the physiological demands associated with this sport. $^{5}$ It is important to understand the physiological competitive sporting requirements, ${ }^{6,7}$ as studied in able-bodied sports, to ensure that training reflects the demands of the sport. For this reason, heart rate (HR) monitors are mainly used to determine the exercise intensity of a training session or a competition in WB. ${ }^{5,6,8,9}$ In addition, many studies are available on the metabolic and cardiovascular responses to wheelchair and arm ergometry, ${ }^{10}$ but despite attempts to improve and optimize current training methods and sport-specific training, ${ }^{5}$ more studies are required focused on the physiological demands of WB in specific training tasks.

Small-sided games (SSGs) are specific training tasks with the goal of reducing interactions and increasing the ratio of players' participation in decision-making but preserving basic variability properties from the game. ${ }^{11}$ That is why, nowadays, SSGs are becoming increasingly popular in many team sports such as soccer, ${ }^{3,4}$ rugby, ${ }^{12}$ Australian football ${ }^{13}$ and specially in basketball. ${ }^{14,15}$ Essentially, SSGs represent a highly intense game, either specific or non-specific to the sport, which focuses on some aspect that the coach is attempting to improve. ${ }^{16}$ The supposition that SSGs may simulate the physiological workloads and intensities commensurate with actual match play while also developing technical and tactical proficiency has led to its popularity as a training modality in the applied and scientific domain within recent years. Moreover, SSGs appear to replicate movement demands and require players to make decisions under pressure and in conditions of fatigue. ${ }^{3}$ Nevertheless, only one study has analyzed the physiological responses in SSGs in WB. ${ }^{17}$

In many of the papers cited above, HR was a commonly used tool to describe physiological responses during matches ${ }^{1,6,8}$ and training tasks. ${ }^{17}$ However, HR was analyzed in absolute values instead of in relative values or according to the percentage of the time spent in each HR zone. Furthermore, none of these studies analyzed the differences between players with and without spinal cord injury (SCI). In addition, some studies cast doubt on the validity of HR measurements in athletes with $\mathrm{SCI} ;{ }^{18,19}$ therefore, it could be interesting to analyze other ways of quantifying WB demands, such as rating of perceived exertion (RPE). RPE also forms a basis for prescribing an intensity of work paralleling that measured by physiological standards of HR, respiratory metabolic functions and lactate production. ${ }^{18}$ For this reason, some authors ${ }^{20}$ proposed differentiated RPE in comparison with the overall measure, to better explain the mechanisms of the subjective perceived exertion that can determine physical work. They distinguished three rates of RPE, focused on (i) 'local' or 'muscle' (RPEmus, that is, the feeling of strain in the working muscles),

Faculty of Education and Sport, University of the Basque Country, Vitoria-Gasteiz, Spain

Correspondence: A Iturricastillo, Faculty of Education and Sport, University of the Basque Country, UPV/EHU, Lasarte 71, 01007 Vitoria-Gasteiz, Spain.

E-mail: aitor.iturricastillo@ehu.eus

Received 11 November 2015; revised 7 March 2016; accepted 7 March 2016; published online 12 July 2016 
Table 1 Wheelchair basketball players' characteristics with (SCI) and without (Non-SCI) spinal cord injury

\begin{tabular}{|c|c|c|c|c|c|c|c|}
\hline Player & Group & Injury & Age (years) & IWBF classification & AIS grade & Injury time (years) & Training experience (years) \\
\hline P1 & $\mathrm{SCl}$ & Spina Bifida (L1) & 16 & 1 & A & 16 & 2 \\
\hline P2 & & Spinal cord injury (T1-T2) & 36 & 1 & A & 34 & 20 \\
\hline P3 & & Spinal cord injury (incomplete (C5-C6) & 35 & 3 & C & 30 & 18 \\
\hline P4 & & Spinal cord injury (T10) & 30 & 3 & A & 2 & 1 \\
\hline P5 & & Spinal cord injury (T12-L3) & 42 & 1 & A & 18 & 7 \\
\hline P6 & & Spinal cord injury (T3) & 19 & 1 & A & 12 & 2 \\
\hline Sample $(n=6)$ & & & $30 \pm 10$ & - & - & $18.67 \pm 11.78$ & $8.33 \pm 8.54$ \\
\hline P7 & Non-SCl & Osteoarthritis congenital & 40 & 4 & & 40 & 21 \\
\hline P8 & & Hip labral tear & 18 & 4 & & 2 & 2 \\
\hline P9 & & Knee injury & 25 & 4,5 & & 5 & 2 \\
\hline P10 & & Viral disease (polio) & 35 & 3,5 & & 33 & 4 \\
\hline P11 & & Knee injury & 41 & 4,5 & & 9 & 9 \\
\hline P12 & & Amputation & 35 & 4 & & 28 & 15 \\
\hline Sample $(n=6)$ & & & $32 \pm 9$ & - & & $19.17 \pm 15.82$ & $8.83 \pm 7.78$ \\
\hline
\end{tabular}

Abbreviations: AIS, ASIA Impairment Scale; IWBF, International Wheelchair Basketball Federation; SCI, spinal cord injury.

Results are in means \pm s.d.

(ii) 'central' or 'respiratory' (RPEres, that is, perceived tachycardia, tachypnea and even dyspnea) and (iii) 'overall' (RPE overall). Many studies involving people with disability have studied the differentiated RPE method, ${ }^{21-23}$ using laboratory environments, but recently RPE (RPEres and RPEmus) has also been analyzed in WB matches. ${ }^{7}$ Thus, it could be interesting to study the differentiated RPE method in intermittent high-intensity training tasks such as SSGs.

Therefore, the first aim of this study was to examine the physiological responses in commonly used basketball SSGs (4 vs 4) in well-experienced WB players with and without SCI. The second aim was to examine the development of the physiological variables during bouts and compare this evolution between groups.

\section{METHODS}

\section{Participants}

Twelve Spanish First Division WB players (age $30.8 \pm 9.5$ years, sitting body height $82.9 \pm 9.1 \mathrm{~cm}$, body mass $75.7 \pm 10.4 \mathrm{~kg}$, HRmax $177.9 \pm 13.7$ beats per $\mathrm{min})$ participated in the study. The team was divided into two groups according to their injury type (Table 1). On the one hand, the group SCI $(n=6$, sitting body height $82.1 \pm 5.9 \mathrm{~cm}$, body mass $72.4 \pm 8.8 \mathrm{~kg}$, HRmax $173.5 \pm 15.3$ beats per min) and on the other hand, the group Non-SCI ( $n=6$, sitting body height $88.8 \pm 2.0 \mathrm{~cm}$, body mass $78.9 \pm 11.5 \mathrm{~kg}$, HRmax $182.3 \pm 11.5$ beats per min). The participants were classified according to the Classification Committee of the International Wheelchair Basketball Federation (IWBF) (from class $1=$ players with low functional capacity to class $4.5=$ players with high functional capacity). The inclusion criteria for the participants in the study were to have a valid license from the Spanish Federation of Sports for people with Physical Disabilities (FEDDF) and the certificate of disability that is necessary to belong to this federation. The Ethics Committee of the University of the Basque Country approved the study, and all participants provided written informed consent as outlined in the Declaration of Helsinki (2013).

\section{Procedures}

This study was conducted over five consecutive weeks (from November to December) during the competitive period with the team training twice per week (Tuesday and Thursday) and competing at weekends. The SSGs were part of the training sessions; hence, data were collected during the five consecutive Tuesdays, at the same time of the day (8-9 PM) and with at least $48 \mathrm{~h}$ rest between sessions. All the players took part in at least $70 \%$ of the bouts during five consecutive weeks. Thus, a total of 144 individual observations met all requirements and were included in the analysis. Moreover, the analysis took into account all the players who had completed the full training task $(4 \times 4$ minutes). These bouts were presented as bouts 1 to 4 . No strenuous exercises were performed within the $48 \mathrm{~h}$ immediately prior to the training sessions, and the researchers supervised the study at all times.

\section{Data collection}

Endurance test. To obtain the individual maximal HR (HRmax) of each player, all players completed a modified $(10 \mathrm{~m})$ Yo Yo intermittent recovery test level 1 (YYIR1 $10 \mathrm{~m})^{17,24} 1$ week before the SSGs were conducted. The YYIR1 $10 \mathrm{~m}$ consists of repeated $2 \times 10 \mathrm{~m}$ propels at a progressively increased speed controlled by audio bleeps from a pre-recorded source. This endurance test showed good reproducibility values (intraclass correlation coefficient $=$ 0.83-0.94). All players were familiar with the field-testing procedures, as they were part of their usual fitness assessment program. During the test, HR was continuously monitored at 1-s intervals by telemetry (Polar Team Sport System, Polar Electro Oy, Kempele, Finland). The HRmax was determined from the highest value from the YYIR1.

Small-sided games (SSGs). The SSGs were performed on a basketball pitch $(15 \times 28 \mathrm{~m})$ with a duration $(4 \times 4 \mathrm{~min}$ separated by $2 \mathrm{~min}$ of passive recovery) that was strictly controlled and has already been implemented by other researchers. ${ }^{17}$ The game rules were the same as in a competitive match, with the exception of no free throws after a fault or time-outs allowed during the 4-min periods to avoid excessive stops. During the SSGs, two supporting subjects were located out of the play area with several balls for immediate availability to minimize any disruption of play, and thus the total duration of the SSGs represented the effective time of exercise. The teams were balanced with respect to their disability and according to the IWBF functional classification. Thus, players with SCI were intermixed with players without SCI to balance both groups. All the SSGs were preceded by a 10-min standardized warm-up ( 3 min of aerobic activity without the ball plus two linear sprints and two sprints with a change of direction). Players were not allowed to consume any type of drinks during the recovery periods, and all players received verbal encouragement from the coaches.

Heart rate during SSGs. HR was continuously monitored throughout the SSGs at 1-s intervals by telemetry (Polar Team Sport System, Polar Electro Oy). The HRmean and HRpeak were recorded in absolute values for all bouts of the SSGs. The HRmean expressed relative to each players' HRmax (\%HRmax) for the entire $4 \times 4$ min SSG section of each training session was used for analysis. ${ }^{14}$ In addition, four HR zones were established, and the percentage of the time spent in each zone during SSGs was calculated. The HR zones were defined as low ( $<75 \%$ of HRmax), moderate ( $75-85 \%$ of HRmax), high $(85-95 \%$ of HRmax) and maximal ( $>95 \%$ of HRmax), according to previously established criteria in basketball. ${ }^{1,15}$ The average value and standard deviation (s.d.) of each bout and group were used for the statistical analysis. 
Table 2 Physiological responses between players with ( $\mathrm{SCl}$ ) and without (Non-SCI) spinal cord injury in WB 4 vs 4 small-sided games

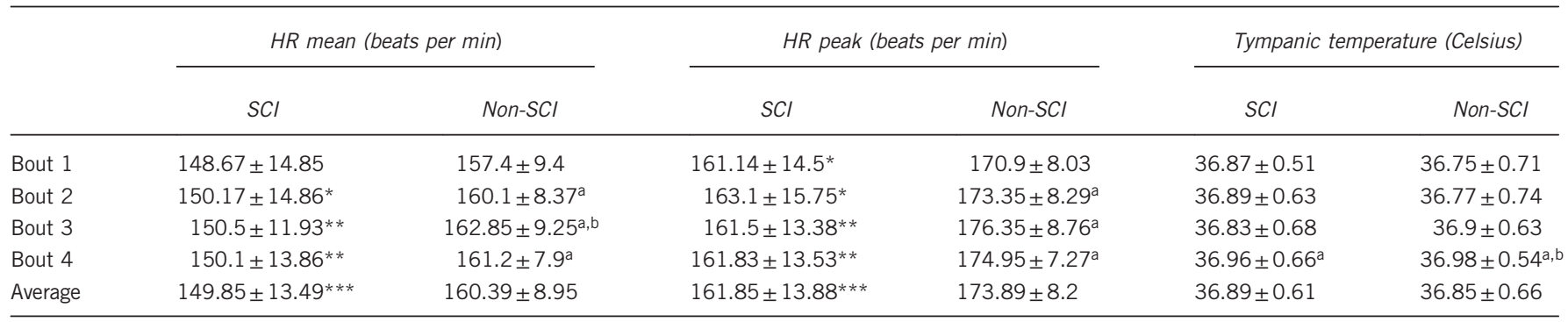

Abbreviations: $\mathrm{HR}$, heart rate; $\mathrm{SCl}$, spinal cord injury; WB, wheelchair basketball.

Values are means $( \pm$ s.d. $) .{ }^{*} P<0.05,{ }^{* *} P<0.01,{ }^{* * *} P<0.001$ : significant difference between $\mathrm{SCI}$ and Non-SCl players.

asignificant difference $(P<0.05)$ compared with bout 1

bignificant difference $(P<0.05)$ compared with bout 2 .

Tympanic temperature. A ThermoScan 5 IRT 4520 (Braun GmbH, Kronberg, Germany) was used to measure tympanic temperature. This is an infrared thermometer used in the predictive mode that can provide a measurement in seconds using an algorithm that extrapolates from the speed at which temperature changes as the thermometer warms up. ${ }^{17,25}$ The data were recorded before the warm-up and immediately at the end of each 4-min bout by the same investigator on all occasions. The average value and s.d. of each bout and group were used for the statistical analysis.

Perceived effort. The 10-point scale proposed by Foster et al. ${ }^{26}$ was used immediately after each SSG bout to determine how hard the exercise had been. Participants responded separately about the RPEres and the arm muscular RPEmus. ${ }^{22-24}$ The same investigator recorded the data on all occasions, immediately after each 4-min bout. Each player completed the RPE scale without the presence of other players, and they could not see the values of other participants. Players were educated about the 10-point scale during a month before the data collection. Moreover, this scale was used in all the previous training sessions. The average value and s.d. of each bout and group were used for the statistical analysis.

\section{Data analysis}

Data analysis was performed using the Statistical Package for Social Sciences (version 20.0 for Windows, SPSS, Chicago, IL, USA). Standard statistical methods were used for the calculation of the mean and standard deviations. Data were screened for normality of distribution and homogeneity of variances using a Shapiro-Wilk normality test. Student's $t$-test for independent samples was used to determine the differences between groups in physiological responses in each bout independently, and analysis of variance repeated measures with an appropriate Bonferroni post hoc test was used to compare results among bouts in each group (SCI and Non-SCI) independently. In addition, the between-groups comparison from baseline to different bouts was calculated with a two-way mixed analysis of variance (bout $\times$ group). The $P<0.05$ criterion was used to establish statistical significance.

\section{RESULTS}

The absolute mean values of the physiological responses (HRmean, HRpeak and tympanic temperature) in a 4 vs 4 SSG are presented in Table 2. The Non-SCI group showed significantly higher values than the SCI group in HRmean and HRpeak but not in tympanic temperature. In addition, significant differences were observed between the first and the other bouts in HRmean and HRpeak in the Non-SCI group but not in the SCI group. Regarding tympanic temperature, the final bouts showed differences with respect to the first bouts in both groups. According to the two-way analysis of variance (bout $\times$ group), no variable showed statistical significance.

In relation to HRmean and HRpeak relative values (\%HRmax), non-significant differences were observed between both groups (SCI vs Non-SCI), in all bouts. However, significant differences were observed
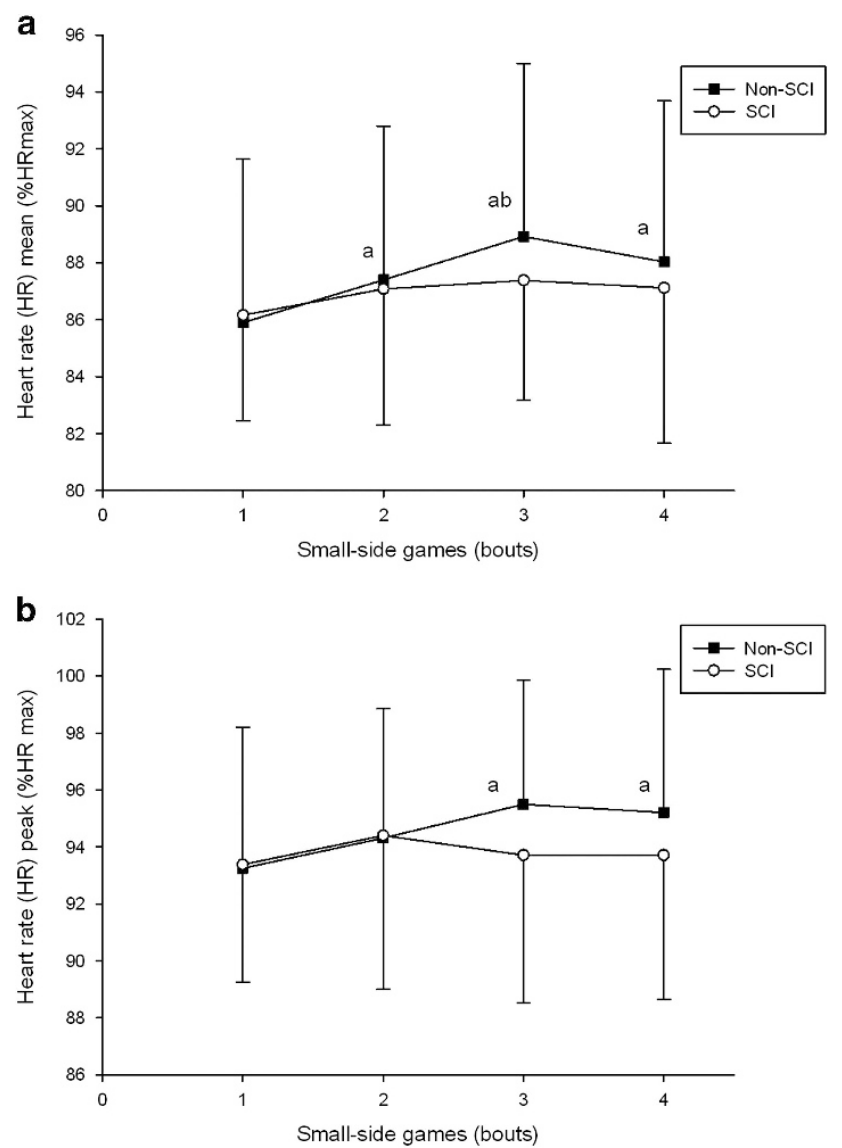

Figure 1 Relative values (\%HRmax) bout to bout for players with $\mathrm{SCl}$ and without $\mathrm{SCl}$ (Non-SCl) in HRmean (a) and HRpeak (b). asignificant differences $(P<0.05)$ compared with bout 1 , bSignificant differences $(P<0.05)$ compared with bout 2 .

in the Non-SCI group among bouts in HRmean (Figure 1a) and HRpeak (Figure 1b) but not in the SCI group.

Similarly, when intensity of exercise was expressed as a percentage of the time spent in different HR zones of HRmax, non-significant differences were observed between the SCI and the Non-SCI groups (Table 3). With respect to the differences among bouts in the Non-SCI group, the percentage of the time spent at $75-85 \%$ was significantly lower in the third and fourth bouts compared with the first one. In addition, significantly higher values were observed in the Non-SCI group in the second and third bouts compared with the first in the percentage of time spent at more than $95 \%$ of HRmax. 
Table 3 The percentage of the time spent in different heart rate (HR) zones between players with (SCI) and without (Non-SCl) spinal cord injury in wheelchair basketball 4 vs 4 small-sided games

\begin{tabular}{|c|c|c|c|c|c|}
\hline HR zones (\%HRmax) & Group & Bout 1 & Bout 2 & Bout 3 & Bout 4 \\
\hline \multirow[t]{2}{*}{ Time at $<75 \%$} & $\mathrm{SCl}$ & $7.77 \pm 7.83$ & $4.3 \pm 3.56$ & $3.85 \pm 4.23$ & $4.86 \pm 7.14$ \\
\hline & Non-SCl & $8.3 \pm 8.54$ & $6.72 \pm 6.24$ & $7.91 \pm 10.1$ & $7.87 \pm 12.17$ \\
\hline \multirow[t]{2}{*}{ Time at $75-85 \%$} & $\mathrm{SCl}$ & $29.82 \pm 27.08$ & $35.89 \pm 29.54$ & $28.98 \pm 26.05$ & $27.32 \pm 26.22$ \\
\hline & Non-SCl & $35.95 \pm 28.13$ & $27.64 \pm 25.5$ & $20.18 \pm 16.89^{a}$ & $24.08 \pm 19.23^{a}$ \\
\hline \multirow[t]{2}{*}{ Time at $85-95 \%$} & $\mathrm{SCl}$ & $53.33 \pm 25.13$ & $44.02 \pm 24.29$ & $53.15 \pm 23.98$ & $50.03 \pm 27.22$ \\
\hline & Non-SCl & $38.62 \pm 21.24$ & $43.97 \pm 23.04$ & $45.43 \pm 25.19$ & $44.9 \pm 23.92$ \\
\hline \multirow[t]{2}{*}{ Time at >95\% } & $\mathrm{SCl}$ & $9.65 \pm 16.35$ & $15.83 \pm 24.63$ & $14.06 \pm 20.22$ & $17.74 \pm 24.45$ \\
\hline & Non-SCl & $15.74 \pm 25.25$ & $21.25 \pm 31.60^{a}$ & $26.38 \pm 32.83^{a}$ & $23.1 \pm 27.02$ \\
\hline
\end{tabular}

Abbreviations: $\mathrm{HR}$, heart rate; $\mathrm{SCl}$, spinal cord injury. Values are means $( \pm$ s.d. $)$

asignificant difference $(P<0.05)$ compared with bout 1 .
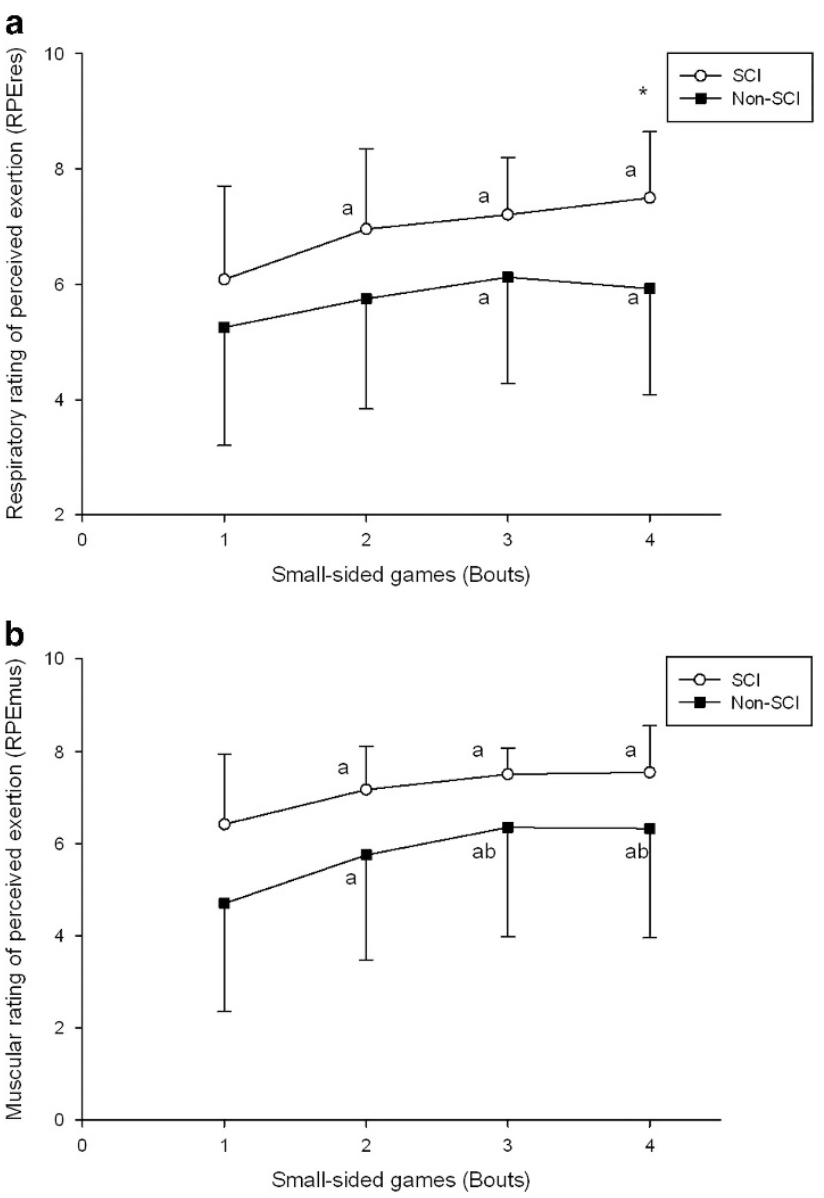

Figure 2 Respiratory (a) and muscular (b) perceived exertion bout to bout for players with $\mathrm{SCl}$ and without $\mathrm{SCl}$ (Non-SCl). *Significant differences $(P<0.05)$ between $\mathrm{SCl}$ and Non-SCl. asignificant differences $(P<0.05)$ compared with bout 1 , bSignificant differences $(P<0.05)$ compared with bout 2 .

The SCI group showed significantly higher values in RPEres in the last bout, but no significant differences were shown in RPEmus (Figure 2). Moreover, for the Non-SCI group in RPEres (Figure 2a), there were significantly higher values in the third and fourth bouts compared with the first one. Regarding RPEmus (Figure 2b), significantly higher values were observed in all bouts compared with the first one and in the third and fourth bouts compared with the second one for both groups. In relation to the SCI group, there were significant differences in all bouts compared with the first one.

\section{DISCUSSION}

In this study, we examined the HR responses, tympanic temperature and perceived exertion in 4 vs 4 SSG. Our results show that the players with SCI and without SCI may have similar HR relative values (\%HRmean, \%HRpeak, percentage of the time spent in different HR zones), although the Non-SCI group attained higher absolute HR (HRmean and HRpeak) values. Furthermore, no differences were observed between the two groups in relation to tympanic temperature. However, the players with SCI reported significantly higher values in RPEres in the last bout than the Non-SCI group for the same SSG.

Several studies have investigated the use of HR in able-bodied players as an indicator of exercise intensity; ${ }^{1,14,15}$ however, there are few works in reference to wheelchair sports. Croft et al. ${ }^{6}$ showed an average HRmean of $146 \pm 16$ beats per min in wheelchair tennis and $163 \pm 11$ beats per min in WB players during a match. Generally, our results are lower than those observed by Croft et al. ${ }^{7}$ but almost the same or higher than those reported by others in the literature (range 128-151 beats per min, respectively) ${ }^{5,8,9}$ for both groups (SCI and Non-SCI). The players with SCI attained a significantly lower HRmean $(149.85 \pm 13.49$ beats per min vs $160.39 \pm 8.95$ beats per $\min )$ and HRpeak ( $161.85 \pm 13.88$ beats per min vs $173.89 \pm 8.2$ beats per min) than the Non-SCI group. Nevertheless, the s.d.s of HRmean and HRmax were higher than those observed in the Non-SCI group. In the case of SCI group, the results were higher than those reported by Goosey et al. ${ }^{27}$ probably owing to the diversity in the lesion level of the SCI group of our study. It is well known that the work capacity of individuals with SCI is limited by loss of functional muscle mass and sympathetic control. Sympathetic nervous system impairment limits control of regional blood flow and cardiac output, and HRmax following cervical lesions may be reduced to $110-130$ beats per min. ${ }^{27}$ Thus, our results suggest that absolute $\mathrm{HR}$ values have to be interpreted with caution in players with SCI during high-intensity training tasks as they can lead to error.

Some authors reported absolute HR values in relation to a competitive WB match, ${ }^{5,8,9}$ but only one study reported relative HRmean values $(83.9 \pm 1.9 \%){ }^{6}$ In this sense, both groups (SCI and Non-SCI) reached similar relative HRmean values (\% HRmax) to those reported by Croft et al. ${ }^{6}$ during WB matches ( $\mathrm{SCI}=86.16 \pm 3.7 \%$; Non-SCI $=85.9 \pm 5.73 \%$ ). Thus, to improve physical capacities, SSG could be useful because they represent highly intense games that simulate real play situations. Despite significant differences being observed in absolute values of HRmean and HRpeak between players 
with and without SCI during the 4 vs 4 SSG, there were none in the relative values (\%HRmean, \%HRpeak, percentage of the time spent in different HR zones). However, the SSG might be good training tasks for WB players because of the similarities with the physiological responses involved in a match. This could be an interesting finding as both groups may train at the same relative intensity, in spite of the difficulty of players with SCI to reach the HRmax. This means that when the values are expressed in relative values of their HRmax, the differences in the physiological demands of SSG for players with and without SCI disappeared. For this reason, relative HR could have more applicability for coaches or physical trainers to quantify the training load instead of absolute HR values.

The training for physiological responses during matches has been analyzed by objective (means of HR) and subjective (RPE) methods. ${ }^{7,17}$ However, there is controversy in the literature about the relationship of HR and RPE for people with SCI. ${ }^{18,19,23}$ Jacobs et al. ${ }^{19}$ found a strong linear association between $\mathrm{HR}$ and $\mathrm{VO}_{2}$ in participants with paraplegia but not between HR and RPE during functional neuromuscular stimulation-assisted ambulation. The results are consistent with those of Lewis et al. ${ }^{18}$ who concluded that RPE is an invalid indicator of exercise intensity in participants with paraplegia. In contrast, evidence suggests that the RPE is effective in controlling moderate $\left(50 \% \mathrm{VO}_{2}\right.$ peak) and vigorous $\left(70 \% \mathrm{VO}_{2}\right.$ peak) intensities during hand cycling exercise in participants with $\mathrm{SCI} .^{23}$ Considering that most studies were conducted in a laboratory setting at a given intensity or in incremental tests instead of field testing, it would be interesting to assess the HR-RPE relationship during the game itself ${ }^{8}$ and specific training tasks. The most intriguing findings of the study were the contrasting tendencies of RPEres values with respect to relative HR values in players with SCI, thus supporting the controversy. However, for the Non-SCI group, there was a similar tendency in HR and RPE, as they showed a similar rising trend in RPE values when increasing absolute and relative HR during bouts. In this sense, the intermittent high-intensity nature and characteristics of the SSG, as well as physiological impairments or diverse functional ability, could be the reason for the contrasting tendencies between HR and RPE values. More studies are therefore necessary to analyze the objective and subjective values in players with SCI during highintensity intermittent training tasks because both methods might be measuring a different internal load during SSG.

It is well accepted that people with SCI have a lesser ability to regulate core temperature because of impaired vasomotor and sudomotor activity below their level of injury. ${ }^{28}$ In this case, nonsignificant differences were observed between the SCI and Non-SCI groups in 4 vs 4 full court SSG, regarding tympanic temperature. This might be due to the short duration of the exercise, despite it being at high intensity. Nevertheless, in both groups, significantly higher values were observed between the first and the last bout. Furthermore, the Non-SCI group showed significantly higher values in the last bout with respect to the second bout. Core temperature has an important role in physical performance, ${ }^{29}$ given that it could be adversely affected by the increase in core temperature at rest and during exercise. ${ }^{30}$ If adequate fluid intake is not maintained, the players' thermoregulatory capacity is diminished. ${ }^{31}$ In individuals with SCI, this situation may become a potential physiological problem, as a lack of sympathetic vasomotor adjustment and reduced sweating capacity below the lesion level may hamper appropriate blood redistribution and limit cooling efficiency. ${ }^{32}$

\section{Practical applications}

The main conclusion of this study is that players with and without SCI may have similar HR relative values, although the Non-SCI group attained higher absolute HR values. Therefore, it would be interesting to analyze not only the absolute values of HR but the relative values during WB to quantify the intensity and the internal load of SCI players. For future studies, it would be interesting to compare the relative HR values between SCI and Non-SCI players in different training tasks or matches to determine whether the training or match intensity is the same for every player or not. On the other hand, the players with SCI reported significantly higher values in respiratory RPE in the last bout than the Non-SCI group for the same SSG. Thus, by means of SSG, we might be able to develop anaerobic capacity in a similar manner both in the Non-SCI group and the SCI group. However, the contrasting tendencies between HR and RPE values lead us to reflect about the need for more studies in players with SCI as both methods (HR and RPE) might be measuring a different internal load during SSG.

\section{DATA ARCHIVING}

There were no data to deposit.

\section{CONFLICT OF INTEREST}

The authors declare no conflict of interest.

\section{ACKNOWLEDGEMENTS}

We thank the players and coaches of the wheelchair basketball team CD Zuzenak for facilitating data collection and for the opportunity to carry out this investigation. The present study was financed by a grant from the Basque Government awarded to Aitor Iturricastillo with reference number PRE_2015_2_0262.

1 Ben Abdelkrim N, El Fazaa S, El Ati J. Time-motion analysis and physiological data of elite under-19-year-old basketball players during competition. Br J Sports Med 2007; 41: 69-75.

2 Scanlan AT, Dascombe BJ, Reaburn P, Dalbo VJ. The physiological and activity demands experienced by Australian female basketball players during competition. J Sci Med Sport 2012; 15: 341-347.

3 Hill-Haas SV, Dawson B, Impellizzeri FM, Coutts AJ. Physiology of small-sided games training in football: a systematic review. Sports Med 2011; 41: 199-220.

4 Köklü Y, Aşçi A, Koçak FU, Alemdaroğlu U, Dündar U. Comparison of the physiological responses to different small-sided games in elite young soccer players. J Strength Cond Res 2011; 25: 1522-1528.

5 Bloxham LA, Bell GJ, Bhambhani Y, Steadward RD. Time motion analysis and physiological profile of Canadian world cup wheelchair basketball players. Sports Med 2001; 10: 183-198.

6 Croft L, Dybrus S, Lenton J, Goosey-Tolfrey V. A comparison of the physiological demands of wheelchair basketball and wheelchair tennis. Int J Sports Physiol Perform 2010; 5: 301-315.

7 Iturricastillo A, Yanci J, Granados C, Goosey-Tolfrey VL. Quantifying wheelchair basketball match load: a comparison of heart rate and perceived exertion methods. Int J Sports Physiol Perform 2015 (in press).

8 Coutts KD. Heart rates of participants in wheelchair sports. Paraplegia 1988; 26: 43-49.

9 Schmid A, Huonker M, Stober P, Barturen JM, Schimdt-Trucksäss A, Dürr H et al. Physical performance and cardiovascular and metabolic adaptation of elite female wheelchair basketball players in wheelchair ergometry and in competition. Am J Phys Med Rehabil 1998; 77: 527-533.

10 Aguiar M, Botelho G, Lago C, Maças V, Sampaio J. A review on the effects of soccer small-sided games. J Hum Kinetics 2012; 33: 103-113.

11 Vanlandewijck YC, Daly DJ, Theisen DM. Field test evaluation of aerobic, anaerobic, and wheelchair basketball skill performances. Int J Sports Med 1999; 20: 548-554.

12 Foster CD, Twist C, Lamb KL, Nicholas CW. Heart rate responses to small-sided games among elite junior rugby league players. J Strength Cond Res 2010; 24: 906-911.

13 Davies MJ, Young W, Farrow D, Bahnert A. Comparison of agility demands of small-sided games in elite Australian football. Int J Sports Physiol Perform 2013; 8: 139-147.

14 Sampaio J, Abrantes C, Leite N. Power, heart rate and perceived exertion responses to $3 \times 3$ and 4x4 basketball small-sided. Rev Psícología del Deporte 2009; 18: 463-467. 
15 Delextrat A, Kraiem S. Heart-rate responses by playing position during ball drills in basketball. Int J Sports Physiol Perform 2013; 8: 410-418.

16 Hoffmann JJ, Reed JP, Leiting K, Chiang CY, Stone M. Repeated sprints, high intensity interval training, small sided games: theory and application to field sports. Int J Sports Physiol Perform 2014; 9: 352-357.

17 Yanci J, Iturricastillo A, Granados C. Heart rate and body temperature responses of wheelchair basketball players in small-sided games. Int J Perform Anal Sport 2014; 14: 535-544.

18 Lewis JE, Nash MS, Hamm LF, Martins SC, Groah SL. The relationship between perceived exertion and physiologic indicators of stress during graded arm exercise in persons with spinal cord injuries. Arch Phys Med Rehabil 2007; 88 1205-1211.

19 Jacobs PL, Klose KJ, Guest R, Needham-Shropshire B, Broton JG, Green BA. Relationships of oxygen uptake, heart rate, and ratings of perceived exertion in persons with paraplegia during functional neuromuscular stimulation assisted ambulation. Spinal Cord 1997; 35: 292-298.

20 Ekblom B, Goldbarg AN. The influence of physical training and other factors on the subjective rating of perceived exertion. Acta Physiol Scand 1971; 83: 399-406.

21 Goosey-Tolfrey V, Lenton J, Goddard J, Oldfield V, Tolfrey K, Eston R. Regulating intensity using perceived exertion in spinal cord injured participants. Med Sci Sports Exerc 2010; 42: 608-613.

22 Al-Rahamneh H, Faulkner J, Byrne C, Eston R. Relationship between perceived exertion and physiologic markers during arm exercise with able-bodied participants and participants with poliomyelitis. Arch Phys Med Rehabil 2010; 91: 273-277.
23 Paulson TA, Bishop NC, Leicht CA, Goosey-Tolfrey VL. Perceived exertion as a tool to self-regulate exercise in individuals with tetraplegia. Eur J Appl Physiol 2013; 113 201-209.

24 Granados C, Yanci J, Badiola A, Iturricastillo A, Otero M, Olasagasti J et al. Anthropometry and performance in wheelchair basketball. J Strength Cond Res 2015; 29: 1812-1820.

25 Hamilton PA, Marcos LS, Secic M. Performance of infrared ear and forehead thermometers: a comparative study in 205 febrile and afebrile children. J Clin Nurs 2013; 22: 2509-2518.

26 Foster C, Florhaug JA, Franklin J, Gottschall L, Hrovatin LA, Parker S et al. A new approach to monitoring exercise training. J Strength Cond Res 2001; 15: 109-115.

27 Goosey-Tolfrey VL, Leicht CA. Field-based physiological testing of wheelchair athletes. Sports Med 2013; 43: 77-91.

28 Trbovich M, Ortega C, Schroeder J, Fredrickson M. Effect of a cooling vest on core temperature in athletes with and without spinal cord injury. Top Spinal Cord Inj Rehabil 2014; 20: 70-80.

29 West DJ, Cook CJ, Beaven M, Kilduff LP. The influence of the time of day on core temperature and lower body power output in elite rugby union sevens players. J Strength Cond Res 2014; 28: 1524-1528.

30 Bhambhani Y. Physiology of wheelchair racing in athletes with spinal cord injury. Sports Med 2002; 32: 23-51.

31 Jung AP, Bishop PA, AI-Nawwas A, Dale RB. Influence of hydration and electrolyte supplementation on incidence and time to onset of exercise associated muscle cramps. J Athl Train 2005; 40: 71-75.

32 Theisen D, Vanlandewijck Y. Cardiovascular responses and thermoregulation in individuals with spinal cord injury. Eur Bull Adapt Phys Act 2002; 1: 1-21. 